The rational points of a definable set

Pila, J. and Wilkie, A. J.

2006

MIMS EPrint: 2007.198

Manchester Institute for Mathematical Sciences

School of Mathematics

The University of Manchester

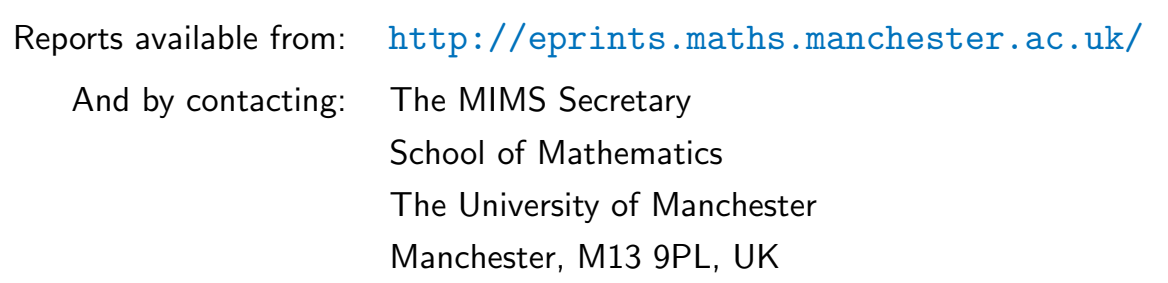

ISSN 1749-9097 


\title{
The rational points of a definable set
}

\author{
J. Pila and A. J. Wilkie
}

\begin{abstract}
Let $X \subset \mathbb{R}^{n}$ be a set that is definable in an o-minimal structure over $\mathbb{R}$. This paper shows that, in a suitable sense, there are very few rational points of $X$ that do not lie on some connected semialgebraic subset of $X$ of positive dimension.
\end{abstract}

2000 Mathematics Subject Classification: 11G99, 03C64

\section{Introduction}

This paper is concerned with the distribution of rational and integer points on certain nonalgebraic sets in $\mathbb{R}^{n}$. To contextualize the kind of results sought, and in particular to motivate the present setting of definable sets in o-minimal structures over $\mathbb{R}$ (see 1.7 below), we begin by describing earlier results.

The ideas pursued here grew from the paper [4] of Bombieri and the first author, where a technique using elementary real-variable methods and elementary algebraic geometry was used to establish upper bounds for the number of integer points on the graphs of functions $y=f(x)$, under various natural smoothness and convexity hypotheses. Results were obtained for $f$ variously assumed to be (sufficiently) smooth, algebraic, or real-analytic. Several results concerned the homothetic dilation of a fixed $X: y=f(x)$.

1.1. Definition. Let $X \subset \mathbb{R}^{n}$. For a real number $t \geq 1$ (which will always be tacitly assumed), the homothetic dilation of $X$ by $t$ is the set $t X=\left\{\left\langle t x_{1}, \ldots, t x_{n}\right\rangle:\left\langle x_{1}, \ldots, x_{n}\right\rangle \in X\right\}$. By $X(\mathbb{Z})$ we denote the subset of $X$ comprising the points with integer coordinates.

Suppose now that $X$ is the graph of a function $f:[0,1] \rightarrow \mathbb{R}$. Trivially, one has $\#(t X)(\mathbb{Z}) \leq t+1$ (with equality for e.g. $f(x)=x$ and positive integral $t$ ). According to Jarnik [14], a strictly convex arc $\Gamma: y=g(x)$ of length $\ell$ contains at most $3(4 \pi)^{-1 / 3} \ell^{2 / 3}+O\left(\ell^{1 / 3}\right)$ integer points (and moreover the exponent and constant are best possible). So if $X$ is strictly convex, one infers that

$$
\#(t X)(\mathbb{Z}) \leq c(X) t^{2 / 3} .
$$

However, Swinnerton-Dyer [31] showed that a substantially better estimate may be obtained if $f$ is assumed $C^{3}$ and strictly convex, namely

$$
\#(t X)(\mathbb{Z}) \leq c(X, \epsilon) t^{3 / 5+\epsilon}
$$

for all positive $\epsilon$. (Regarding this circle of "limited smoothness" problems see also [29, 20].)

Counting integer points on $t X$ is of course the same as counting points $\langle m / t, n / t\rangle$ on $X$, and in this guise such questions arose in work of Sarnak [27] on betti numbers of abelian covers. He conjectured that if $f$ is $C^{\infty}$ and strictly convex then in fact

$$
\#(t X)(\mathbb{Z}) \leq c(X, \epsilon) t^{1 / 2+\epsilon}
$$

for all positive $\epsilon$. The exponent $1 / 2$ is best possible here in view of $f(x)=x^{2}$. This conjecture was the starting point of [4], where it is affirmed. 
If $f$ is assumed transcendental analytic, however, then the exponent may be reduced to $\epsilon$, and this result of [4] is the prototype for the results to be presented here.

1.2. Theorem. ([4, Theorem 1]) Let $f:[0,1] \rightarrow \mathbb{R}$ be a transcendental real-analytic function. Let $X$ be the graph of $f$, and let $\epsilon>0$. There is a constant $c(X, \epsilon)$ such that

$$
\#(t X)(\mathbb{Z}) \leq c(X, \epsilon) t^{\epsilon} \cdot \square
$$

Theorem 1.2 answered a question also raised by Sarnak [27], and has found application in that context $[19,28]$. It can be adapted to a result on rational points of bounded height, which we proceed to state.

1.3. Definition. Let $H: \mathbb{Q} \rightarrow \mathbb{R}$ denote the usual height function: $H(a / b)=\max (|a|, b)$ where $a, b \in \mathbb{Z}, b>0, \operatorname{gcd}(a, b)=1$. Extend to $H: \mathbb{Q}^{n} \rightarrow \mathbb{R}$ by setting $H\left\langle\alpha_{1}, \ldots, \alpha_{n}\right\rangle=$ $\max \left(H\left(\alpha_{j}\right)\right)$. If $X \subset \mathbb{R}^{n}$, let $X(\mathbb{Q})$ denote the subset of points with rational coordinates. Set (for $T \geq 1$ )

$$
X(\mathbb{Q}, T)=\{P \in X(\mathbb{Q}), H(P) \leq T\}
$$

and define the density function of $X$ to be

$$
N(X, T)=\# X(\mathbb{Q}, T)
$$

This is not the usual projective height $H_{\text {proj }}$, which is not especially natural in the context of non-algebraic sets, although this makes no difference to our results. The density function $N(X, T)$ is a natural function to study in situations where $X(\mathbb{Q})$ may be infinite, and is the primary object of study here. The aim is to establish upper bounds for $N(X, T)$ under natural geometric conditions on $X$, and with the guiding idea that transcendental sets should contain "few" rational points, in an appropriate sense.

1.4. Theorem. ([20, Theorem 9]) Let $f:[0,1] \rightarrow \mathbb{R}$ be a transcendental analytic function with graph $X$ and $\epsilon>0$. There is a constant $c(X, \epsilon)$ such that

$$
N(X, T) \leq c(X, \epsilon) T^{\epsilon}
$$

Now if $f$ is of special form (e.g. $f(x)=e^{x}$, or, say, a $G$-function) then one may have much stronger results (or at least conjectures) on the scarcity of rational (or even algebraic) points (see e.g. [1]). At the other extreme, constructions going back to Weierstrass (see e.g. [18, 25]) show that an entire transcendental $f$ may take rational values at every rational argument. These constructions do not take much care of the height density of points.

However, given any function $\epsilon:[1, \infty) \rightarrow \mathbb{R}$, strictly decreasing with $\epsilon(t) \rightarrow 0$ as $t \rightarrow \infty$, it is possible (see $[22,7.5]$ ) to construct a transcendental analytic function $f$ on $[0,1]$ and a (rather lacunary) sequence of positive integers $T_{j} \rightarrow \infty$ such that

$$
N\left(X, T_{j}\right) \geq T_{j}^{\epsilon\left(T_{j}\right)}
$$

Thus the above result cannot be much improved in general. (E.g. taking $\epsilon(t)=(\log t)^{-1 / 2}$ shows that for certain $X$ no bound of form $N(X, T) \leq C(\log T)^{K}$ holds etc.) 
Let us now turn to higher dimensional sets. The idea of applying the methods of [4] to the density function of a higher dimensional transcendental analytic set - e.g. the graph $X$ of a transcendental analytic function $f:[0,1]^{2} \rightarrow \mathbb{R}-$ was prompted by a question of Bourgain (see [22]). Those methods entail studying the intersections of $X$ with algebraic hypersurfaces of high degree. Such intersections may be highly singular, though they are still semianalytic. One is further led to consider images under projections: and thus one is led to the class of (bounded) subanalytic sets ([3]) as the natural (and more general) class of sets to consider. The fundamental properties of subanalytic sets: Uniformization theorem, Gabrielov's theorem, and Tamm's theorem (see [3]), enabled the first author to establish analogues of 1.2 and 1.4 for compact subanalytic sets of dimension 2 in $\mathbb{R}^{n}$.

To frame this result, it is necessary to consider a new feature that arises for sets $X$ of dimension 2 and higher: Namely, $X$ may contain semialgebraic subsets of positive dimension, even if $X$ itself is not semialgebraic, and these semialgebraic subsets may contain "many", i.e. $>>T^{\delta}$ for some positive $\delta$, rational points of height $\leq T$. For example, if $X=\{\langle x, y, z\rangle: z=$ $\left.x^{y}, x, y \in[1,2]\right\}$ then each rational $y$ gives rise to a semialgebraic curve in $X$. To accommodate this feature, we make the following definition.

1.5. Definition. Let $X \subset \mathbb{R}^{n}$. The algebraic part of $X$, denoted $X^{\text {alg }}$, is the union of all connected semialgebraic subsets of $X$ of positive dimension. The transcendental part of $X$ is the complement $X-X^{\text {alg }}$.

The algebraic part of a set may be hard to identify in general, and it may be a complicated set: in the above example it consists of infinitely many connected components (one for each $y \in \mathbb{Q} \cap[1,2]$ ), and so is not subanalytic: this point is discussed further below. However, if the algebraic part is excluded from the count of rational points, a result of the quality as 1.4 may be obtained.

1.6. Theorem ([23, Theorem 1.1]) Let $X \subset \mathbb{R}^{n}$ be a compact subanalytic set of dimension 2 and let $\epsilon>0$. There is a constant $c(X, \epsilon)$ such that

$$
N\left(X-X^{\mathrm{alg}}, T\right) \leq c(X, \epsilon) T^{\epsilon}
$$

The corresponding result for integer points on the dilation of such a set is in [22], where 1.2 and 1.4 are also "upgraded" to apply to any 1 dimensional compact subanalytic set $X \subset \mathbb{R}^{n}$.

The exclusion of $X^{\text {alg }}$, where rational points may well accumulate, is weakly analogous to (and suggested by) the notion of the special set in diophantine geometry, and the philosophy of "Geometry Governs Arithmetic" [13, $\S$ F.5]. The conjecture of Lang ([16, Ch I $\S 3$; or 13, $\S$ F.5]) asserts that an algebraic variety $V$ is mordellic (i.e. has only finitely many rational points) outside its special set. When $V$ is a curve, this is the Mordell conjecture, proved by Faltings. In higher dimensions it remains very much open. The special set is the union of non-constant images of projective spaces and elliptic curves. Excluding the algebraic part from our count is much coarser, but seems to be the appropriate way of separating out the essentially transcendental part of the problem.

For results on integer points on surfaces, and higher dimensional hypersurfaces, under hypotheses of a differential-geometric nature see [2, 29, 30]. 
The generalization of 1.6 to arbitrary compact subanalytic sets in $\mathbb{R}^{n}$ was conjectured in [22]. Such sets are globally subanalytic (meaning subanalytic as subsets of the compact real analytic manifold $\mathbb{P}^{n}(\mathbb{R})$ ). The class of globally subanalytic sets is an example of an o-minimal structure, and this turns out to be the natural setting for our arguments.

The notion of an o-minimal structure arose in the study of model-theoretic questions about $\mathbb{R}$ ("Tarski's problem"; [6]). Here is a definition (following [34]; however, readers will need to be familiar with the theory as developed e.g. in [7] to follow our proofs).

1.7. Definition. A pre-structure is a sequence $\mathcal{S}=\left(S_{n}: n \geq 1\right)$ where each $\mathcal{S}_{n}$ is a collection of subsets of $\mathbb{R}^{n}$. A pre-structure $\mathcal{S}$ is called a structure (over the real field) if, for all $n, m \geq 1$, the following conditions are satisfied:

(1) $\mathcal{S}_{n}$ is a boolean algebra (under the usual set-theoretic operations)

(2) $\mathcal{S}_{n}$ contains every semi-algebraic subset of $\mathbb{R}^{n}$

(3) if $A \in \mathcal{S}_{n}$ and $B \in \mathcal{S}_{m}$ then $A \times B \in \mathcal{S}_{n+m}$

(4) if $m \geq n$ and $A \in \mathcal{S}_{m}$ then $\pi[A] \in \mathcal{S}_{n}$, where $\pi: \mathbb{R}^{m} \rightarrow \mathbb{R}^{n}$ is projection onto the first $n$ coordinates

If $\mathcal{S}$ is a structure and $X \subset \mathbb{R}^{n}$, we say $X$ is definable in $\mathcal{S}$ if $X \in \mathcal{S}_{n}$. If $\mathcal{S}$ is a structure and, in addition,

(5) the boundary of every set in $\mathcal{S}_{1}$ is finite

then $\mathcal{S}$ is called an o-minimal structure (over the real field).

The paradigm example of an o-minimal structure is provided by the semialgebraic sets: closure under projections is the content of the Tarski-Seidenberg theorem [7].

The class of semi-analytic sets is not closed under (proper) projections (see the classical example due to Osgood in [3]). But, as mentioned above, the globally subanalytic sets form an o-minimal structure, denoted $\mathbb{R}_{\text {an }}$ : It is the closure under complementation that is hardest to establish: The abovementioned theorem of Gabrielov is the key to his proof of this fact [10].

Another example of an o-minimal structure is the structure $\mathbb{R}_{\text {exp }}$ "generated by" $e^{x}$. Here $\mathcal{S}_{n}$ is the collection of subsets of $\mathbb{R}^{n}$ of the form $X=\pi\left(f^{-1}(0)\right)$ where, for some $m \geq n, f$ : $\mathbb{R}^{m} \rightarrow \mathbb{R}$ is an exponential polynomial (i.e. $f\left(x_{1}, \ldots, x_{m}\right)=Q\left(x_{1}, \ldots, x_{m}, e^{x_{1}}, \ldots, e^{x_{m}}\right)$ for some polynomial $Q \in \mathbb{R}\left[X_{1}, \ldots, X_{2 m}\right]$ ), and where $\pi: \mathbb{R}^{m} \rightarrow \mathbb{R}^{n}$ is projection on the first $n$ coordinates. The requisite finiteness property (5) follows from Khovanskii's theorem [15]. In showing that $\mathbb{R}_{\exp }$ is a structure, the major difficulty is again the "theorem of the complement", which was established by the second author in [33]. Note that $\mathbb{R}_{\exp }$ contains e.g. ([9]) sets such as $\left\{\left\langle x, x^{r}\right\rangle, x>0\right\}$ for positive irrational $r$, or $\left\{\left\langle x, e^{-1 / x}\right\rangle, x>0\right\}$ which do not belong to $\mathbb{R}_{\mathrm{an}}$ (they are not subanalytic at the origin).

In fact the structure $\mathbb{R}_{\text {an,exp }}$ generated by the union of $\mathbb{R}_{\text {an }}$ and $\mathbb{R}_{\text {exp }}$ is o-minimal [8]. However it is known [26] that there is no "largest" o-minimal structure, and that there are o-minimal structures containing nowhere analytic functions.

Thus o-minimal structures provide rich and flexible categories to work in, while at the same time a natural setting for our methods.

Let then $\mathcal{S}$ be an o-minimal structure (over $\mathbb{R}$ ). A definable set $X \subset \mathbb{R}^{n}$ will mean a set definable in $\mathcal{S}$. Now we can state a preliminary version of our main result. 
1.8. Theorem. (First version) Let $X \subset \mathbb{R}^{n}$ be a definable set, and $\epsilon>0$. There is a constant $c(X, \epsilon)$ such that

$$
N\left(X-X^{\text {alg }}, T\right) \leq c(X, \epsilon) T^{\epsilon}
$$

The proof of the theorem begins by showing that the points in question reside on "few" (i.e. $O_{X, \epsilon}\left(T^{\epsilon}\right)$ ) hypersurfaces of suitable degree $d(\epsilon)$; it then proceeds by induction on the dimension of $X$. Thus it is necessary to have an estimate of the same form as above for those hypersurface intersections but in which the implied constant is uniform over all intersections of $X$ with hypersurfaces of fixed degree: i.e., a result for a definable family of sets. The following convention will be adopted. In considering subsets $Z=\{\langle x, y\rangle\} \subset \mathbb{R}^{n} \times \mathbb{R}^{m}$, projection on the first factor will be denoted $\pi_{1}$, and on the second $\pi_{2}$. Put $Y=Y_{Z}=\pi_{2}(Z)$ and for $y \in Y$ put $Z_{y}=\left\{z \in Z: \pi_{2}(z)=y\right\}$, and $X_{y}=X_{Z, y}=\pi_{1}\left(Z_{y}\right)$ its image in $\mathbb{R}^{n}$. A family $Z \subset \mathbb{R}^{n} \times \mathbb{R}^{m}$ of sets will mean the collection of fibres $\left\{X_{y}: y \in Y_{Z}\right\}$. A family $Z$ is definable if the set $Z$ is. The result to be proved is then the following.

1.9. Theorem. (Second version) Let $Z \subset \mathbb{R}^{n} \times \mathbb{R}^{m}$ be a definable family, and $\epsilon>0$. There is a constant $c(Z, \epsilon)$ with the following property. Let $X$ be a fibre of $Z$. Then

$$
N\left(X-X^{\mathrm{alg}}, T\right) \leq c(Z, \epsilon) T^{\epsilon}
$$

The example $X=\left\{\langle x, y, z\rangle \in \mathbb{R}^{3}: z=x^{y}, x, y \in[2,3]\right\}$, for which $X^{\text {alg }}=\{\langle x, y, z\rangle \in$ $X: y \in \mathbb{Q}\}$, shows that $X^{\text {alg }}$ is not, in general, semialgebraic (or even definable: a definable set has only finitely many connected components). Nevertheless, it might be supposed that, for any $X$ and $\epsilon$, there is a semialgebraic set $X_{\epsilon} \subset X$ and a constant $c(X, \epsilon)$ such that $N\left(X-X_{\epsilon}, T\right) \leq$ $c(X, \epsilon) T^{\epsilon}$. This is not the case: Consider $X=\left\{\langle x, y\rangle: 0<x<1,0<y<e^{x}\right\}$. Then $X^{\text {alg }}=X$ but $X$ is not semialgebraic (otherwise its bounding graph $y=e^{x}, x \in[0,1]$ would be semialgebraic). So $N\left(X-X_{\epsilon}, T\right)>>T^{4}$ for any semialgebraic $X_{\epsilon} \subset X$. However, it is possible to find a definable $X_{\epsilon} \subset X^{\text {alg }}$ with the desired property; indeed, for a definable family $Z$ the sets $X_{\epsilon}$ may be taken to be fibres of a definable family $W(Z, \epsilon) \subset Z$, and this is the final version of the result to be proved.

1.10. Theorem. (Final version) Let $Z \subset \mathbb{R}^{n} \times \mathbb{R}^{m}$ be a definable family and $\epsilon>0$. There is a definable family $W=W(Z, \epsilon) \subset Z$ and a constant $c(Z, \epsilon)$ with the following property. Let $y \in Y$. Put $X=X_{Z, y}$ and $X_{\epsilon}=X_{W, y}$. Then $X_{\epsilon} \subset X^{\text {alg }}$ and

$$
N\left(X-X_{\epsilon}, T\right) \leq c(Z, \epsilon) T^{\epsilon}
$$

Note that this version makes a nontrivial assertion in situations, like the example above, in which $\left(X-X^{\text {alg }}\right)(\mathbb{Q})$ is empty but $X^{\text {alg }}$ is not definable.

The diophantine part of the proof follows the strategy going back to [4]. The heart of the analytic part of the proof is the possibility of a certain uniform parameterization of the fibres $X$ in a definable family. The uniformity required is in the number of $C^{(r)}$ maps $(0,1)^{\operatorname{dim}(X)} \rightarrow X$ required to cover $X$, and at the same time in bounds on the sizes of all their partial derivatives up to some prescribed finite order $r$. This is achieved in $\S 2-5$, by establishing an o-minimal version 
of Gromov's Algebraic Reparameterization Lemma (see [11, page 232]; itself a refinement of a method of Yomdin [36, 37]) for obtaining such parameterizations of closed semialgebraic sets.) Our o-minimal version of Gromov's Reparameterization Lemma may well find other applications.

(Note added: Gromov's proof is very brief; a careful proof has been given by Burguet [5].)

In [22] a conjecture is also made about integer points on the dilation of a compact subanalytic set. That conjecture is essentially (though not strictly) weaker than the corresponding statement about rational points, and is also affirmed here, for (bounded) definable sets, in $\S 8$. Other results on integer points on definable curves are obtained in [35].

While, as indicated, the estimate $N\left(X-X^{\text {alg }}, T\right)=O_{X, \epsilon}\left(T^{\epsilon}\right)$ cannot be improved for globally subanalytic sets, a much better estimate might be anticipated for other o-minimal structures where we have more control over the definable sets. For example:

1.11. Conjecture. Let $X$ be definable in $\mathbb{R}_{\text {exp }}$. Then there are constants $c_{1}(X), c_{2}(X)$ such that (for $T \geq e$ )

$$
N\left(X-X^{\mathrm{alg}}, T\right) \leq c_{1}(X)(\log T)^{c_{2}(X)} .
$$

It should be observed that the results here give, even in the original situation of one dimensional sets $X: y=f(x)$ in $\mathbb{R}^{2}$, an extension of the previous results: Curves definable in o-minimal structures may have derivatives that degenerate at an endpoint, or they may be not analytic at any point (e.g. the examples in [26]). The previous results may be inapplicable in such situations, and thus the present results may well find further application in the circle of problems $[27,19,28]$ that provided their original motivation.

In this paper, $A \subset B$ means that $A$ is a subset of (possibly equal to) $B$. The cardinality of a set $A$ is denoted $\# A$, and $\mathbb{N}$ denotes the set of nonnegative integers. The letters $i, j, k, \ell, m, n, r, d$ are reserved exclusively to range over $\mathbb{N}$.

\section{Acknowledgements}

The authors are very grateful to the referees for their most helpful comments and suggestions. The first author thanks A. Venkatesh and L. van den Dries for assistance. He is grateful to the Mathematical Institute, Oxford, and in particular A. Lauder and R. Heath-Brown, for hospitality during a visit in which parts of this paper were written. His work was partly supported by McGill University and a grant from NSERC. The second author is grateful to all the staff at, and visitors to, the Isaac Newton Institute for Mathematical Sciences for making the Programme on Model Theory and Applications to Algebra and Analysis (January 17 -July 15 2005) so successful. The contents of this paper were first presented there, and he thanks, in particular, Zoe Chatzidakis and Sergei Starchenko for extremely helpful comments.

\section{Reparameterization (after Yomdin-Gromov)}

For $\S 2-5$ we fix an o-minimal structure $\mathcal{S}$ over a real closed field $M$; definable will mean definable in $\mathcal{S}$ (see [7]). Although we are ultimately only interested in $\mathbb{R}$, the greater generality actually simplifies the arguments here because it guarantees a certain "uniformity in parameters" that would be absent if we restricted our attention to structures over $\mathbb{R}$. 
Recall that an element $a \in M$ is called finite if $|a| \leq c$ for some $c \in \mathbb{N}$ (we assume that $\mathbb{Q}$ is identified with the prime subfield of $M$ ). A finite element of $M$ will also be called strongly bounded. An $n$-tuple of elements of $M$ is strongly bounded if all its coordinates are, and a definable subset of $M^{n}$ is strongly bounded if there is a fixed finite bound for all the coordinates of all its elements. Further, a definable function is strongly bounded if its graph is (equivalently, if its domain and range are).

2.1. Definition. Let $X \subset M^{n}$ be definable. A definable function $\phi:(0,1)^{\ell} \rightarrow X$, where $\ell=\operatorname{dim} X$, is called a partial parameterization of $X$. A finite set $S$ of partial parametrizations of $X$ is called a parameterization of $X$ if $\cup_{\phi \in S}$ range $(\phi)=X$. (Of course standard notation like “ $(0,1)$ " refers to its natural interpretation in $M$.)

We shall be interested in various extra conditions on the functions in such an $S$. In particular, it is not hard to show, using the $C^{(r)}$-cell decomposition theorem ([7]), that every bounded set has a $C^{(r)}$-parameterization. We shall be interested in bounding the derivatives.

2.2. Definition. A parameterization $S$ (of some definable set $X$ ) is called an $r$-parameterization if every $\phi \in S$ is of class $C^{(r)}$ and has the property that $\phi^{(\alpha)}$ is strongly bounded for each $\alpha \in \mathbb{N}^{\operatorname{dim} X}$ with $|\alpha| \leq r$, where $|\alpha|$ is the sum of the coordinates of $\alpha$.

2.3. Theorem. (Reparametrization Theorem [after Gromov]) For any $r \in \mathbb{N}$ and any strongly bounded, definable set $X$, there exists an r-parameterization of $X$.

There is also a version for functions.

2.4. Definition. Suppose that $S$ is an $r$-parameterization of the definable set $X \subset M^{m}$ and that $F: X \rightarrow M^{n}$ is a definable function. Then we say that $S$ is an $r$-reparameterization of $F$ if, for each $\phi \in S, F \circ \phi$ is of class $C^{(r)}$ and $(F \circ \phi)^{(\alpha)}$ is strongly bounded for all $\alpha \in \mathbb{N}^{\operatorname{dim} X}$ with $|\alpha| \leq r$.

2.5. Theorem. For any $r \in \mathbb{N}$ and any strongly bounded, definable function $F$, there exists an $r$-reparameterization of $F$.

The next 3 sections are devoted to the proof of theorems 2.3 and 2.5. It will be convenient to assume that $M$ is $\aleph_{0}$-saturated, in particular non-archimedean, in these sections. This can always be assumed by taking a suitable elementary extension and noting that the statements of 2.3 and 2.5 pull back to the original structure.

\section{The unary function case}

There is a very simple, but crucial, analytic trick at the heart of the proof of 2.5 which we now state and prove. Indeed, the rest of the argument is just a case of organizing the induction carefully.

3.1. Lemma. Let $r \geq 2$ and suppose that $f:(0,1) \rightarrow M$ is a definable function of class $C^{(r)}$ with $f^{(j)}$ strongly bounded for $0 \leq j \leq r-1$. Suppose further that $\left|f^{(r)}\right|$ is (weakly) decreasing. Define $g:(0,1) \rightarrow M$ by

$$
g(x)=f\left(x^{2}\right)
$$


Then $g^{(j)}$ is strongly bounded for $0 \leq j \leq r$.

Proof. By the chain rule (applied in $M), g^{(i)}(x)=\sum_{j=0}^{i} \rho_{i, j}(x) \cdot f^{(j)}\left(x^{2}\right)$, for each $i=$ $0,1, \ldots, r$ and $x \in(0,1)$, where each $\rho_{i, j}$ is a polynomial with integer coefficients (of degree $j$, in fact).

Now, by our hypothesis on $f$, all summands are strongly bounded except, possibly, the one with $i=j=r$. One easily checks that this summand is $2^{r} x^{r} f^{(r)}\left(x^{2}\right)$. Let $c$ be a positive integer strongly bounding the function $f^{(r-1)}$ and suppose, for a contradiction, that there is a some $x_{0} \in(0,1)$ with $\left|f^{(r)}\left(x_{0}\right)\right|>4 c / x_{0}$. By the Mean Value Theorem (applied in $M$ ), there is some $\xi \in\left[x_{0} / 2, x_{0}\right]$ such that $f^{(r-1)}\left(x_{0}\right)-f^{(r-1)}\left(x_{0} / 2\right)=f^{(r)}(\xi) .\left(x_{0}-x_{0} / 2\right)$. But by our hypothesis on $f^{(r)}$ we have $\left|f^{(r)}(\xi)\right| \geq\left|f^{(r)}\left(x_{0}\right)\right|>4 c / x_{0}$. Hence

$$
2 c \geq\left|f^{(r-1)}\left(x_{0}\right)-f^{(r-1)}\left(x_{0} / 2\right)\right|>\frac{4 c}{x_{0}}\left(x_{0}-x_{0} / 2\right)=2 c .
$$

This contradiction shows that

$$
\left|2^{r} x^{r} f^{(r)}\left(x^{2}\right)\right| \leq 2^{r} x^{r} \frac{4 c}{x^{2}}
$$

for all $x \in(0,1)$, and the right-hand side here is bounded by $2^{r+2} c$ since $r \geq 2$. Thus $g^{(i)}$ is strongly bounded for $i=0,1, \ldots, r$, and the lemma is proved.

3.2. Lemma. Let $F:(0,1) \rightarrow M$ be a definable, strongly bounded function. Then $F$ has a 1-reparameterization, $S$ say, with the additional property that, for each $\phi \in S$, either $\phi$ or $F \circ \phi$ is a polynomial (restricted to $(0,1)$ ) with strongly bounded coefficients.

Proof. By o-minimality, choose elements $a_{0}=0<a_{1}<\ldots<a_{p}<a_{p+1}=1$ of $M$ so that, for each $i=0,1, \ldots, p, F$ is of class $C^{(1)}$ and satisfies either $\left|F^{\prime}\right| \leq 1$ throughout $\left(a_{i}, a_{i+1}\right)$ or $\left|F^{\prime}\right| \geq 1$ throughout $\left(a_{i}, a_{i+1}\right)$.

In the first case, define $\phi_{i}:(0,1) \rightarrow M$ by $x \mapsto\left(a_{i+1}-a_{i}\right) x+a_{i}$.

In the second case (when $F$ is certainly strictly monotone and continuous on $\left(a_{i}, a_{i+1}\right)$ ) we set $b_{i}=\lim _{x \rightarrow a_{i}^{+}} F(x), b_{i+1}=\lim _{x \rightarrow a_{i+1}^{-}} F(x)$ and define $\phi_{i}:(0,1) \rightarrow M$ by $x \mapsto$ $F^{-1}\left(\left(b_{i+1}-b_{i}\right) x+b_{i}\right)$.

In either case, range $\left(\phi_{i}\right)=\left(a_{i}, a_{i+1}\right)$ and both $\phi_{i}$ and $F \circ \phi_{i}$ are of class $C^{(1)}$ throughout $(0,1)$ with strongly bounded derivatives. Further, at least one of these functions is linear with coefficients in $[-1,1]$. It is now clear that $S=\left\{\phi_{0}, \phi_{1}, \ldots, \phi_{p}, \hat{a_{1}}, \ldots, \hat{a_{p}}\right\}$ is a 1 reparameterization of $F$ with the required additional property, where each $\hat{a_{i}}$ denotes the constant function on $(0,1)$ with value $a_{i}$. $\square$

3.3. Lemma. Let $r \geq 1$ and suppose that $F:(0,1) \rightarrow M$ is a definable, strongly bounded function. Then $F$ has an r-reparameterization (with the additional property that, for each $\phi$ in it, either $\phi$ or $F \circ \phi$ is a polynomial (restricted to $(0,1)$ ) with strongly bounded coefficients).

Proof. The proof (of the whole statement, including the parenthetical property) is by induction on $r$. The case $r=1$ being Lemma 3.2, suppose that $r \geq 2$ and that $S$ is an $(r-1)$ reparameterization of $F$ with the additional property. Let $\phi \in S$ and write $\{\phi, F \circ \phi\}=\{g, h\}$ 
where $g$ is a polynomial (restricted to $(0,1)$ ) with strongly bounded coefficients. Thus, in particular, $g^{(i)}$ exists and is strongly bounded for all $i$. However, we only know that $h^{(i)}$ exists, is continuous, and is strongly bounded for $i=0, \ldots, r-1$. In order to apply Lemma 3.1 we use o-minimality to pick elements $0=a_{0}<a_{1}<\ldots<a_{p_{\phi}}<a_{p_{\phi}+1}=1$ in $M$ (depending on $\phi$ ) so that, for each $i=0, \ldots, p_{\phi}$, the function $h$ is of class $C^{(r)}$ on $\left(a_{i}, a_{i+1}\right)$ and $\left|h^{(r)}\right|$ is (weakly) monotonic on $\left(a_{i}, a_{i+1}\right)$.

Let $\theta_{\phi, i}:(0,1) \rightarrow(0,1)$ be defined by

$$
\theta_{\phi, i}(x)= \begin{cases}\left(a_{i+1}-a_{i}\right) x+a_{i}, & \text { if }\left|h^{(r)}\right| \text { is (weakly) decreasing } \\ \left(a_{i}-a_{i+1}\right) x+a_{i+1}, & \text { if }\left|h^{(r)}\right| \text { is (weakly) increasing }\end{cases}
$$

(We choose the first option, say, if $\left|h^{(r)}\right|$ is constant.)

It is immediate from the inductive hypothesis that $h \circ \theta_{\phi, i}:(0,1) \rightarrow M$ is of class $C^{(r)}$, and that $\left(h \circ \theta_{\phi, i}\right)^{(i)}$ is strongly bounded for $i=0, \ldots, r-1$. Further, $\left|\left(h \circ \theta_{\phi, i}\right)^{(r)}\right|$ is (weakly) decreasing. Let $\rho:(0,1) \rightarrow(0,1)$ be the $C^{(\infty)}$ bijection sending $x$ to $x^{2}$. Then by Lemma 3.1, the function $h \circ \theta_{\phi, i} \circ \rho:(0,1) \rightarrow M$ has strongly bounded $i$-th derivative for $i=0, \ldots, r$. Of course, the function $g \circ \theta_{\phi, i} \circ \rho$ is still a polynomial with strongly bounded coefficients and $\left\{h \circ \theta_{\phi, i} \circ \rho, g \circ \theta_{\phi, i} \circ \rho\right\}=\left\{\phi \circ \theta_{\phi, i} \circ \rho, F \circ\left(\phi \circ \theta_{\phi, i} \circ \rho\right)\right\}$. Notice also that as $i$ varies from 0 to $p_{\phi}$, range $\left(\phi \circ \theta_{\phi, i} \circ \rho\right)$ covers range $(\phi)$ apart from finitely many points. So we only have to add finitely many constant functions (taking values in $(0,1)$ ) to the set $\left\{\phi \circ \theta_{\phi, i} \circ \rho: \phi \in S\right\}$ in order for it to become an $r$-reparameterization of $F$ with the required additional property. This completes the induction and the proof of the lemma.

3.4. Corollary. Let $X$ be a strongly bounded subset of $M$ and $F: X \rightarrow M$ a strongly bounded function. Then for all $r \geq 1, F$ has an r-reparameterization.

Proof. Since $X$ is a (finite) union of strongly bounded intervals and points, it clearly has an $r$-parameterization, $S$ say, by linear and constant functions. Now use Lemma 3.3 to $r$ reparameterize each funcion $F \circ \phi:(0,1) \rightarrow M$, for $\phi \in S$, and take the union of these reparameterizations.

We now proceed to the case of functions taking values in $M^{n}$ for $n \geq 2$. However, there is nothing special about unary functions in this process, so we do the general case now.

3.5. Lemma. Let $m, r \geq 1$ and assume that every definable, strongly bounded function with domain a subset $X$ of $M^{\ell}$ (for some $\ell \leq m$ ) and range a subset of $M$, has an r-reparameterization. Then for any $n \geq 1$, the same is true for such functions having range a subset of $M^{n}$ (and domain $X)$.

Proof. It is clearly sufficient (by the obvious inductive argument) to show that if $n \geq 2$ and $F: X \rightarrow M^{n-1}, f: X \rightarrow M$ are definable, strongly bounded functions such that $F$ has an $r$-reparameterization, then so does the function $\langle F, f\rangle: X \rightarrow M^{n}$, where we may as well suppose that $X$ is a definable (strongly bounded) subset of $M^{m}$.

So let $S$ be an $r$-reparameterization of $F$, and let $\phi \in S$. Say $\phi:(0,1)^{\ell} \rightarrow X$ where $\ell=\operatorname{dim}(X) \leq m$. Apply the hypothesis of the lemma to the function $f \circ \phi:(0,1)^{\ell} \rightarrow M$, to obtain an $r$-reparameterization of it, $T_{\phi}$ say. Then each $\psi \in T_{\phi}$ has domain $(0,1)^{\ell}$, and it clearly 
follows by repeated use of the Chain Rule that each function $(\phi \circ \psi)^{(\alpha)}:(0,1)^{\ell} \rightarrow M^{m}$, for $\alpha \in \mathbb{N}^{\ell}$ with $|\alpha| \leq r$, is strongly bounded. It is now easy to check that $\left\{\phi \circ \psi: \phi \in S, \psi \in T_{\phi}\right\}$ is an $r$-reparameterization of $\langle F, f\rangle$, as required.

3.6. Corollary. Let $n \geq 1$ and suppose that $F: X \rightarrow M^{n}$ is a strongly bounded function, where $X$ is a (strongly bounded) subset of $M$. Then for any $r \geq 1, F$ has an r-reparameterization.

Proof. This is immediate from Corollary 3.4 and the case $m=1$ of Lemma 3.5. $\square$

\section{Some questions of convergence}

In Gromov's proof things can be arranged, it seems, so that derivatives are a priori bounded, and we need to be able to reduce to this situation. We shall achieve this by first truncating our given function and finding the reparameterization for the truncation. We then let the truncations converge to the original function. So we require an observation that allows us to conclude that the reparameterizations converge as well. In fact, we lose one level of differentiability here, but this will hardly matter. The final proof of Theorems 2.3 and 2.5 are so arranged that we only require a theory of convergence for unary functions, so we only treat that case here.

So suppose that $N \geq 1, N \in \mathbb{N}$, and that $\left\{F_{t}:(0,1) \rightarrow(0,1)^{N}: t \in(0,1)\right\}$ is a definable family of functions (meaning that the map sending $\langle t, x\rangle$ to $F_{t}(x)$ is a definable function on $\left.(0,1)^{2}\right)$. Suppose further that $r \geq 1$, that all the functions $F_{t}$ are of class $C^{(r)}$, and that their derivatives $F_{t}^{(i)}$ are strongly bounded for $i=0, \ldots, r$. Since we are assuming that $M$ is nonarchimedean, this clearly implies a uniform finite bound $c$ say. Using o-minimality we may define a function $F_{0}:(0,1) \rightarrow[0,1]^{N}$ by $F_{0}(x)=\lim _{t \rightarrow 0^{+}} F_{t}(x)$.

Now the fact that $r \geq 1$ implies that $F_{0}$ is continuous. For suppose that $x_{1}, x_{2} \in(0,1)$ are distinct. Choose $t \in(0,1)$ so that $\| F_{0}\left(x_{i}\right)-F_{t}\left(x_{i}\right)|| \leq\left|x_{1}-x_{2}\right|$ for $i=1,2$. (We use the sup norm $\left\|\left\langle u_{1}, \ldots, u_{p}\right\rangle\right\|=\max \left\{\left|u_{1}\right|, \ldots,\left|u_{p}\right|\right\}$ on cartesian products of $M$ throughout.) By the Mean Value Theorem (in $M$ ), we also have that $\left\|F_{t}\left(x_{1}\right)-F_{t}\left(x_{2}\right)\right\| \leq N c\left|x_{1}-x_{2}\right|$ (as $c$ is a bound for $F_{t}^{\prime}(x)$ for $x \in\left[x_{1}, x_{2}\right]$ ). Thus

$$
\begin{gathered}
\left\|F_{0}\left(x_{1}\right)-F_{0}\left(x_{2}\right)\right\| \leq\left\|F_{0}\left(x_{1}\right)-F_{t}\left(x_{1}\right)\right\|+\left\|F_{t}\left(x_{1}\right)-F_{t}\left(x_{2}\right)\right\|+\left\|F_{t}\left(x_{2}\right)-F_{0}\left(x_{2}\right)\right\| \\
\leq(N c+2)\left|x_{1}-x_{2}\right|
\end{gathered}
$$

whence the continuity of $F_{0}$.

One can now go on to show that for each $i=0, \ldots, r-1, F_{0}$ is of class $C^{(i)}, F_{t}^{(i)}$ is strongly bounded and, indeed, that $F_{0}^{(i)}(x)=\lim _{t \rightarrow 0^{+}} F_{t}^{(i)}(x)$ for each $x \in(0,1)$. (This result properly belongs to the theory of "definably Banach" spaces (over o-minimal structures) currently being developed by the second author and Margaret Thomas. The simplest example is the set $\Omega^{(r)}$ of all $M$-definable functions $F:(0,1) \rightarrow M$ with continuous and bounded derivatives up to order $r$, which is naturally a normed vector space for the field structure on $M$ and with norm $\|F\|^{(r)}:=\sup _{x \in(0,1), i=0, \ldots, r}\left|F^{(i)}(x)\right|$. If $\sigma=\left\{F_{t}: t \in(0,1)\right\}$ is a definable family contained in $\Omega^{(r)}$ then it is clear what we should mean by saying that $\sigma$ is Cauchy (as $\left.t \rightarrow 0^{+}\right)$, and it is routine to check that the pointwise limit of $\sigma$ is, indeed, the $\|\cdot\|^{(r)}$-limit and lies in $\Omega^{(r)}$ if $\sigma$ is Cauchy. More important for us here, however, is the fact, borrowed and 
modified from the classical theory, that $\Omega^{(r)}$ is "definably compactly" contained in $\Omega^{(r-1)}$ for all $r \geq 1$. In other words, every $\|\cdot\|^{(r)}$-bounded, definable family $\sigma$ in $\Omega^{(r)}$ is Cauchy in $\Omega^{(r-1)}$, and hence the pointwise limit of $\sigma$ lies in $\Omega^{(r-1)}$. The crucial point in the o-minimal setting is that one knows, a priori, that this limit function is $(r-1)$-times continuously differentiable at all but finitely many $x \in(0,1)$.)

We now consider, for each $t \in(0,1)$, the set $S_{t}$ of co-ordinate functions of $F_{t}$. Let us suppose that it parameterizes $(0,1)$, so that it is an $r$-parameterization of $(0,1)$. We define $S_{0}$ to be the set of functions $\left.\phi\right|_{\phi^{-1}[(0,1)]}$ for $\phi$ a co-ordinate function of $F_{0}$. Then

(A) $\bigcup_{\psi \in S_{0}} \operatorname{range}(\psi)=(0,1) \backslash T$ for some finite set $T \subset(0,1)$. (For otherwise, by ominimality, there would be a non-empty, open subinterval of $(0,1)$ missed by each $\psi \in S_{0}$ and hence missed by each corresponding co-ordinate function $\phi$ of $F_{0}$. But this easily contradicts the facts that each $S_{t}$ parameterizes $(0,1)$ and $\lim _{t \rightarrow 0^{+}} F_{t}(x)=F_{0}(x)$ (for $x \in(0,1)$ ), bearing in mind the fact that as $r \geq 1$, the derivative of each co-ordinate function of $F_{t}$ has a uniform finite bound.)

Notice also that

(B) each function $\psi \in S_{0}$ has domain an open subset of $(0,1)$ (which might have infinite complement in $(0,1))$, is of class $C^{(r-1)}$ and is such that $\psi^{(i)}$ is strongly bounded for $i=0, \ldots, r-1$.

We now apply these remarks to set up the inductive process involved in the proofs of 2.3 and 2.5. We fix $m \geq 1$ in 4.1, 4.2 and 4.3.

\subsection{Notation.}

(1) For $U$ a definable, open subset of $M^{m+1}$, we write $V \subset \subset U$ to mean that $V$ is a definable, open subset of $M^{m+1}$ with $V \subset U$ and $\operatorname{dim}(U \backslash V) \leq m$.

(2) For $\phi:(0,1) \rightarrow M$ a definable function, we define $I_{\phi}:(0,1)^{m+1} \rightarrow(0,1)^{m} \times M$ by $\left\langle x_{1}, \ldots, x_{m}, x_{m+1}\right\rangle \mapsto\left\langle x_{1}, \ldots, x_{m}, \phi\left(x_{m+1}\right)\right\rangle$. If $X \subset M^{m+1}$ and $f: X \rightarrow M^{n}$ are definable, $f_{\phi}$ denotes $f \circ I_{\phi}$ (having domain $I_{\phi}^{-1}[X]$ ).

4.2. Lemma. Suppose that $n \geq 1, U \subset \subset(0,1)^{m+1}$ and that $f: U \rightarrow M^{n}$ is a definable, strongly bounded function. Suppose further that for each $i=1, \ldots, m, \partial f / \partial x_{i}$ exists, is continuous and is strongly bounded (on $U$ ).

Then for each $r \geq 2$, there exists an $(r-1)$-parameterization of a cofinite subset of $(0,1)$, $S$ say, and a set $V \subset \subset U$ such that for each $\phi \in S, I_{\phi}[V] \subset U, f_{\phi}$ is of class $C^{(1)}$ on $V$, and all its first partial derivatives $\partial f_{\phi} / \partial x_{i}, i=1, \ldots, m+1$ are strongly bounded (on $V$ ).

Proof. We treat only the case $n=1$. The general case follows using an argument similar to that in the proof of Lemma 3.5. Our $S$ will be constructed from a certain limit set $S_{0}$ (of a suitable family $S_{t}: t \in(0,1)$ ) as described above. (Notice that properties (A) and (B) are not quite the conditions for an $(r-1)$-parameterization of $(0,1)$, though $(\mathrm{A})$ is precisely what we are asking for here, and (B) can easily be modified by composing with linear functions, as we shall see.)

Now, by o-minimality, let $W \subset \subset U$ be such that $f$ is of class $C^{(1)}$ on $W$ and, for each $t, y \in$ $(0,1)$, let $W_{t}(y)$ denote the set of those $\bar{x} \in(0,1)^{m}$ such that the point $\langle\bar{x}, y\rangle$ is at a distance at 
least $t$ from the set $\left([0,1]^{m} \times\{y\}\right) \backslash W$. It follows that the map $\bar{x} \mapsto\left|\partial f / \partial x_{m+1}(\bar{x}, y)\right|$ is defined and continuous on $W_{t}(y)$ and hence achieves its maximum value at some point $s_{t}(y) \in W_{t}(y)$, provided that this set is non-empty. Since $M$ admits definable Skolem functions it follows that $s$ may be taken to be a definable function in both $t$ and $y$ (taking the value $\langle 1 / 2, \ldots, 1 / 2\rangle$, say, if $W_{t}(y)=\emptyset$ ) and that (in all cases)

$$
\begin{gathered}
\forall t \in(0,1), \forall y \in(0,1), \forall \bar{x} \in W_{t}(y), \text { we have }\left\langle s_{t}(y), y\right\rangle \in W \text { and } \\
\left|\partial f / \partial x_{m+1}\left(s_{t}(y), y\right)\right| \geq\left|\partial f / \partial x_{m+1}(\bar{x}, y)\right| .
\end{gathered}
$$

Now consider the definable family $\left\{g_{t}:(0,1) \rightarrow(0,1)^{m} \times M: t \in(0,1)\right\}$ given by $g_{t}(y):=\left\langle s_{t}(y), f\left(s_{t}(y), y\right)\right\rangle$ (where we give $f$ the value 0 , say, if $\left.\left\langle s_{t}(y), y\right)\right\rangle \notin U$ ), and apply Corollary 3.6 to obtain an $r$-reparameterization, $S_{t}$ say, of $g_{t}$, for each $t \in(0,1)$. Now since we are assuming that $M$ is $\aleph_{0}$-saturated it follows easily (using the fact that $M$ admits definable Skolem functions) that for some $N \in \mathbb{N}, S_{t}$ may be taken as the set of co-ordinate functions of some definable function $F_{t}:(0,1) \rightarrow(0,1)^{N}$, where the family $\left\{F_{t}: t \in(0,1)\right\}$ is also definable. Let $S_{0}$ be the limit, as $t \rightarrow 0^{+}$, of this family as described at the beginning of this section. By splitting the functions in $S_{0}$, we may suppose that they are all either constant or injective and have domains an open subinterval of $(0,1)$. Now throw away the constant functions and compose each remaining function with a suitable injective linear function (with coefficients in $[-1,1])$, thereby arriving at an $(r-1)$-parameterization, $S$ say, of a cofinite subset of $(0,1)$.

Now set $V:=\left((0,1)^{m+1} \backslash \bigcup_{\phi \in S} I_{\phi}^{-1}\left[(0,1)^{m+1} \backslash W\right]\right) \cap U$. (See 4.1(2).)

Then the injectivity (and continuity) of the $\phi$ 's imply that $V \subset \subset U$. Clearly $I_{\phi}[V] \subset$ $W \subset U$ and so, also, the function $f_{\phi}$ is of class $C^{(1)}$ on $V$ (for $\phi \in S$ ). It only remains to show that if $\phi \in S$ and $\left\langle\bar{x}_{0}, y_{0}\right\rangle \in V$, then $\partial f_{\phi} / \partial x_{i}\left(\bar{x}_{0}, y_{0}\right)$ is finite, for $i=1, \ldots, m+1$.

Now since $\left\langle\bar{x}_{0}, \phi\left(y_{0}\right)\right\rangle \in W \subset U$, this is clear (by the lemma hypothesis) for $i=1, \ldots, m$. For the remaining case we note that there is some linear function $\lambda$ (with finite coefficients) and some function $\psi$ in $S_{0}$ (or, rather, a subfunction of a function in $S_{0}$ ) such that $\phi(y)=$ $\psi(\lambda(y))$ (for all $y \in(0,1)$ ), and so it is clearly sufficient to show that if $y_{1} \in \operatorname{dom}(\psi)$, then $\psi^{\prime}\left(y_{1}\right) . \partial f / \partial x_{m+1}\left(\bar{x}_{0}, \psi\left(y_{1}\right)\right)$ is finite, where we also know that $\left\langle\bar{x}_{0}, \psi\left(y_{1}\right)\right\rangle \in W$. Since $W$ is open it follows that

(i) $\bar{x}_{0} \in W_{t}\left(\psi\left(y_{1}\right)\right)$ for all sufficiently small $t \in(0,1)$.

Now by definition of $S_{0}$, there is, for each $t \in(0,1)$, (uniformly) a function $\phi_{t} \in S_{t}$ such that $\lim _{t \rightarrow 0^{+}} \phi_{t}\left(y_{1}\right)=\psi\left(y_{1}\right)$ and (as $\left.r \geq 2\right), \lim _{t \rightarrow 0^{+}} \phi_{t}^{\prime}\left(y_{1}\right)=\psi^{\prime}\left(y_{1}\right)$. Hence

(ii) $\left|\partial f / \partial x_{m+1}\left(\bar{x}_{0}, \psi\left(y_{1}\right)\right)-\partial f / \partial x_{m+1}\left(\bar{x}_{0}, \phi_{t}\left(y_{1}\right)\right)\right| \leq 1$ (and $\left\langle\bar{x}_{0}, \phi_{t}\left(y_{1}\right)\right\rangle \in W$ ) for sufficiently small $t \in(0,1)$ (by the continuity of $\partial f / \partial x_{m+1}$ on $W$ ), and

(iii) $\left|\phi_{t}^{\prime}\left(y_{1}\right)-\psi^{\prime}\left(y_{1}\right)\right| \leq\left|\partial f / \partial x_{m+1}\left(\bar{x}_{0}, \psi\left(y_{1}\right)\right)\right|^{-1}$ for sufficiently small $t \in(0,1)$, and

(iv) $\bar{x}_{0} \in W_{t}\left(\phi_{t}\left(y_{1}\right)\right)$ for all sufficiently small $t \in(0,1)$ (since if (i) holds for some $t_{0} \in(0,1)$, then (iv) holds for any $t<t_{0} / 2$ satisfying $\left.\left|\psi\left(y_{1}\right)-\phi_{t}\left(y_{1}\right)\right|<t_{0} / 2\right)$.

Thus, if we select some $t \in(0,1)$ such that (ii)-(iv) all hold simultaneously, we see that

$$
\left.\left|\psi^{\prime}\left(y_{1}\right) \cdot \frac{\partial f}{\partial x_{m+1}}\left(\bar{x}_{0}, \psi\left(y_{1}\right)\right)\right| \leq\left|\phi_{t}^{\prime}\left(y_{1}\right)\right| \cdot\left|\frac{\partial f}{\partial x_{m+1}}\left(\bar{x}_{0}, \psi\left(y_{1}\right)\right)\right|+1, \quad \text { (by (iii) }\right),
$$




$$
\begin{gathered}
\leq\left|\phi_{t}^{\prime}\left(y_{1}\right)\right| \cdot\left|\frac{\partial f}{\partial x_{m+1}}\left(\bar{x}_{0}, \phi_{t}\left(y_{1}\right)\right)\right|+\left|\phi_{t}^{\prime}\left(y_{1}\right)\right|+1, \quad \text { (by (ii)), } \\
\leq\left|\phi_{t}^{\prime}\left(y_{1}\right)\right| \cdot\left|\frac{\partial f}{\partial x_{m+1}}\left(s_{t}\left(\phi_{t}\left(y_{1}\right)\right), \phi_{t}\left(y_{1}\right)\right)\right|+\left|\phi_{t}^{\prime}\left(y_{1}\right)\right|+1, \quad(\text { by (iv) and }(*)) .
\end{gathered}
$$

However, $\left|\phi_{t}^{\prime}\left(y_{1}\right)\right|$ is certainly finite (since $\phi_{t} \in S_{t}$ ), so it suffices to show that

$$
\phi_{t}^{\prime}\left(y_{1}\right) . \partial f / \partial x_{m+1}\left(s_{t}\left(\phi_{t}\left(y_{1}\right)\right), \phi_{t}\left(y_{1}\right)\right)
$$

is finite. But since $S_{t}$ is an $r$-reparameterization of $g_{t}$ it follows that

(v) $\left(s_{t} \circ \phi_{t}\right)^{\prime}\left(y_{1}\right)$ is finite, and

(vi) $\left.(d / d y)\right|_{y=y_{1}} f\left(s_{t} \circ \phi_{t}(y), \phi_{t}(y)\right)$ is finite.

Now by (vi), the quantity

$$
(s \circ \phi)^{\prime}\left(y_{1}\right) \cdot\left\langle\frac{\partial f}{\partial x_{1}}, \ldots, \frac{\partial f}{\partial x_{m}}\right\rangle\left(s_{t}\left(\phi_{t}\left(y_{1}\right)\right), \phi_{t}\left(y_{1}\right)\right)+\phi_{t}^{\prime}\left(y_{1}\right) \cdot \frac{\partial f}{\partial x_{m+1}}\left(s_{t}\left(\phi_{t}\left(y_{1}\right)\right), \phi_{t}\left(y_{1}\right)\right)
$$

is finite. Also the scalar product term here is finite by (v) and the strong boundedness of the functions $\partial f / \partial x_{i}$ (for $i=1, \ldots, m$ ) as given by the Lemma hypothesis. (Note that $\left\langle s_{t}\left(\phi_{t}\left(y_{1}\right)\right), \phi_{t}\left(y_{1}\right)\right\rangle \in W \subset U$ by (iv) and (*).) Hence the second term is finite, which is what we had to show.

4.3. Corollary. Suppose that $r, n \geq 1, U \subset \subset(0,1)^{m+1}$ and that $f: U \rightarrow M^{n}$ is a definable, strongly bounded function. Suppose further that for each $\alpha=\left\langle\alpha_{1}, \ldots, \alpha_{m+1}\right\rangle \in \mathbb{N}^{m+1}$ with $|\alpha| \leq r$ and $\alpha_{m+1}=0, f^{(\alpha)}$ exists, is continuous and is strongly bounded (on $U$ ).

Then for each $k \geq 0$ there exists a set $V_{k} \subset \subset U$ and an r-parameterization of a cofinite subset of $(0,1)$, $S_{k}$ say, such that for each $\phi \in S_{k}, I_{k}\left[V_{k}\right] \subset U, f_{\phi}$ is of class $C^{(r)}$ on $V_{k}$ and all its derivatives $f_{\phi}^{(\alpha)}$ (for $\alpha=\left\langle\alpha_{1}, \ldots, \alpha_{m+1}\right\rangle \in \mathbb{N}^{m+1},|\alpha| \leq r, \alpha_{m+1} \leq k$ ) are strongly bounded (on $\left.V_{k}\right)$.

Proof. We may take $V_{0} \subset \subset U$ such that $f$ is a function of class $C^{(r)}$ on $V_{0}$ (by o-minimality), and $S_{0}=\left\{\left.\mathrm{id}\right|_{(0,1)}\right\}$. So suppose, inductively, that $V_{k}$ and $S_{k}$ have been constructed with the required properties.

Let $\Delta:=\left\{\alpha=\left\langle\alpha_{1}, \ldots, \alpha_{m+1}\right\rangle \in \mathbb{N}^{m+1}:|\alpha| \leq r-1, \alpha_{m+1} \leq k\right\}$, set $\tilde{n}:=\# \Delta \cdot \# S_{k}$, and let $F=\left\langle F_{1}, \ldots, F_{\tilde{n}}\right\rangle: V_{k} \rightarrow M$ be an enumeration of all the functions $f_{\phi}^{(\alpha)}: V_{k} \rightarrow M$ for $\phi \in S_{k}$ and $\alpha \in \Delta$. Then the hypotheses of Lemma 4.2 obtain (with $F$ for $f, V_{k}$ for $U$, $\tilde{n} \cdot n$ for $n$ and $r+1$ in place of $r$ ) - note the " $r-1$ " in the definition of $\Delta-$ so we may choose an $r$-parameterization, $S$ say, of a cofinite subset of $(0,1)$ and a set $V_{k+1} \subset \subset V_{k}$ such that for each $\psi \in S, I_{\psi}\left[V_{k+1}\right] \subset V_{k}$ (so that, in particular $f_{\phi \circ \psi}=\left(f_{\phi}\right)_{\psi}=f_{\phi} \circ I_{\psi}$ is of class $C^{(r)}$ on $V_{k+1}$, being the composition of $C^{(r)}$ functions) and so that each function $\left(f_{\phi}^{(\alpha)}\right)_{\psi}$ is of class $C^{(1)}$ with

(†) $\frac{\partial}{\partial x_{i}}\left(\left(f_{\phi}^{(\alpha)}\right)_{\psi}\right)$ strongly bounded on $V_{k+1}$ for $i=1, \ldots, m+1, \alpha \in \Delta$, and $\phi \in S_{k}$.

Thus, we define

$$
S_{k+1}:=\left\{\phi \circ \psi: \phi \in S_{k}, \psi \in S\right\}
$$


and it remains to show that if $\alpha=\left\langle\alpha_{1}, \ldots, \alpha_{m+1}\right\rangle \in \mathbb{N}^{m+1}$, with $|\alpha| \leq r$ and $\alpha_{m+1} \leq k+1$, and if $\phi \circ \psi \in S_{k+1}$ then $\left(f_{\phi \circ \psi}\right)^{(\alpha)}$ is strongly bounded on $V_{k+1}$.

Now if $\alpha_{m+1}=0$, then this is clear because $\left(f_{\phi \circ \psi}\right)^{(\alpha)}=\left(f_{\phi}^{(\alpha)}\right)_{\psi}$ and $f_{\phi}^{(\alpha)}$ is strongly bounded. If $\alpha_{m+1}>0$, then $\left(f_{\phi \circ \psi}\right)^{(\alpha)}=\partial / \partial x_{m+1}\left(f_{\phi \circ \psi}^{(\beta)}\right)$ for some $\beta \in \Delta$. Further, for $\bar{a}:=\left\langle a_{1}, \ldots, a_{m+1}\right\rangle \in V_{k+1}$,

$$
\left(f_{\phi \circ \psi}\right)^{(\beta)}(\bar{a})=\psi^{\left(\alpha_{m+1}-1\right)}\left(a_{m+1}\right) \cdot\left(f_{\phi}^{(\beta)}\right)_{\psi}(\bar{a}) .
$$

Thus

$$
\left(f_{\phi \circ \psi}\right)^{(\alpha)}(\bar{a})=\psi^{\left(\alpha_{m+1}\right)}\left(a_{m+1}\right) \cdot\left(f_{\phi}^{(\beta)}\right)_{\psi}(\bar{a})+\psi^{\left(\alpha_{m+1}-1\right)}\left(a_{m+1}\right) \cdot \frac{\partial}{\partial x_{m+1}}\left(f_{\phi}^{(\beta)}\right)_{\psi}(\bar{a})
$$

which is finite since we have $\alpha_{m+1} \leq|\alpha| \leq r$ and $\beta \in \Delta$ (see $(\dagger)$ ), and $\psi \in S$, so $\psi^{\left(\alpha_{m+1}-1\right)}$ and $\psi^{\left(\alpha_{m+1}\right)}$ are strongly bounded.

\section{The proofs of 2.3 and 2.5}

For each $m \geq 1$ consider the following two statements.

$(\mathrm{I})_{m}$ For all $r, n \geq 1$ and all definable, strongly bounded functions $F:(0,1)^{m} \rightarrow M^{n}$, there exists an $r$-reparameterization of $F$.

$(\text { II })_{m}$ For all $r \geq 1$, every definable, strongly bounded subset $X \subset M^{m+1}$, there exists an $r$-parameterization of $X$.

Note that $(\mathrm{I})_{1}$ holds by Corollary 3.6. Also, $(\mathrm{II})_{m}$ makes sense for $m=0$ and clearly holds in this case (via linear functions). We proceed by induction to show that the statements hold for all $m \geq 1$. So suppose that $m \geq 1$ and that $(\mathrm{I})_{\ell}$ holds for all $\ell \leq m$ and that (II) holds for all $\ell<m$. We shall show that $(\mathrm{II})_{m}$ holds and then that $(\mathrm{I})_{m+1}$ holds.

For (II) ${ }_{m}$, let $r \geq 1$ and $X \subset M^{m+1}$ be definable and strongly bounded. We may clearly assume that $X$ is a cell in $M^{m+1}$, and we do the more difficult of the two cases, namely $X=(f, g)_{Y}$ where $Y$ is a (strongly bounded) cell in $M^{m}$, and leave the other case, $X=\operatorname{graph}(f \mid Y)$, to the reader.

So let $S$ be an $r$-parameterization of $Y$ (using (II) $)_{m-1}$ ) and for each $\phi \in S$ let $T_{\phi}$ be an $r$-reparameterization of the function $\langle f \circ \phi, g \circ \phi\rangle:(0,1)^{\ell} \rightarrow M^{2}$, where $\ell=\operatorname{dim}(Y)$ (using $\left.(\mathrm{II})_{\ell}\right)$. Then for each $\psi \in T_{\phi}$, define $\theta_{\phi, \psi}:(0,1)^{\ell+1} \rightarrow X$ by

$\theta_{\phi, \psi}(\bar{x}):=\left\langle\phi \circ \psi\left(x_{1}, \ldots, x_{\ell}\right),\left(1-x_{\ell+1}\right) f \circ \phi \circ \psi\left(x_{1}, \ldots, x_{\ell}\right)+x_{\ell+1} g \circ \phi \circ \psi\left(x_{1}, \ldots, x_{\ell}\right)\right\rangle$

where $\bar{x}=\left\langle x_{1}, \ldots, x_{\ell+1}\right\rangle$. Then the set $\left\{\theta_{\phi, \psi}: \phi \in S, \psi \in T_{\phi}\right\}$ is readily seen to be an $r$-parameterization of $X$.

For $(\mathrm{I})_{m+1}$ we need only do the case $n=1$ (by Lemma 3.5), so let $r \geq 1$ and $F$ : $(0,1)^{m+1} \rightarrow M$ be a definable, strongly bounded function. By $(\mathrm{I})_{m}$ there exists, for each $u \in(0,1)$, an $r$-reparameterization, $S_{u}$ say, of the function $F_{u}:(0,1)^{m} \rightarrow M: \bar{x} \mapsto F(\bar{x}, u)$, 
where $\bar{x}=\left\langle x_{1}, \ldots, x_{m}\right\rangle$ and by using a saturation and Skolem function argument (just as in the proof of Lemma 4.2) we may suppose that there exist definable families of functions $\left\{{ }^{(1)} \phi_{u}: u \in(0,1)\right\}, \ldots,\left\{{ }^{(N)} \phi_{u}: u \in(0,1)\right\}$ such that $S_{u}=\left\{{ }^{(1)} \phi_{u}, \ldots,{ }^{(N)} \phi_{u}\right\}$.

Now, for $j=1, \ldots, N$ define the function ${ }^{(j)} F:(0,1)^{m+1} \rightarrow M$ by ${ }^{(j)} F(\bar{x}, u):=$ $F\left({ }^{(j)} \phi(\bar{x}, u), u\right)$. Let

$$
{ }^{*} F:=\left\langle{ }^{(1)} \phi, \ldots,{ }^{(N)} \phi,{ }^{(1)} F, \ldots,{ }^{(N)} F\right\rangle:(0,1)^{m+1} \rightarrow M^{m N+N}
$$

and notice that the hypotheses of Corollary 4.3 hold with ${ }^{*} F$ for $f,(0,1)^{m+1}$ for $U, m N+N$ for $n$. (This is just a restatement of the fact that $S_{u}$ is an $r$-reparameterization of $F_{u}$, uniformly in $u$.) So we apply 4.3 with $k=r$, to obtain $V_{r} \subset \subset(0,1)^{m+1}$ and $S_{r}$ with the properties stated. Now if $V_{r}=(0,1)^{m+1}$ and $S_{r}$ were an $r$-parameterization of all of $(0,1)$, then we could simply take our required $r$-reparameterization of $F$ to consist of the functions ${ }^{(j)} \phi_{\psi}$ for $j=1, \ldots, N$ and $\psi \in S_{r}$. As it is, we at least know that the union of the ranges of these functions (on $(0,1)^{m+1}$ ) covers $(0,1)^{m+1}$ apart from finitely many planes $\left\{x_{m+1}=a\right\}$, and it follows that if we restrict them to the (open) set $V_{r}$ (where they are all of class $C^{(r)}$ and satisfy the bounding condition for $r$-reparameterizability) then they still cover a subset of $(0,1)^{m+1}$ of codimension $\ell$, for some $\ell \leq m$.

Using the (now proven) (II) $)_{m}$, let $T_{1}$ be an $r$-parameterization of $V_{r}$ and $T_{2}$ an $r$ parameterization of the $\ell$-dimensional set $(0,1)^{m+1}-\bigcup_{1 \leq j \leq N}{ }^{(j)} \phi_{\psi}\left[V_{r}\right]$.

For each $\theta \in T_{2}$ we may apply (I) $)_{\ell}$ to obtain an $r$-reparameterization, $U_{\theta}$ say, of the function $F \circ \theta:(0,1)^{\ell} \rightarrow M$. The required $r$-reparameterization of $F$ is now given by

$$
\left\{{ }^{(j)} \phi_{\psi} \circ \chi: j=1 \ldots, N, \psi \in S_{r}, \chi \in T_{1}\right\} \cup\left\{\hat{\theta} \circ \hat{\lambda}: \theta \in T_{2}, \lambda \in U_{\theta}\right\}
$$

where the ${ }^{\wedge}$ denotes extension of the domain of a function from $(0,1)^{\ell}$ to $(0,1)^{m}$ (but leaving its values independent of the last $m-\ell$ variables).

This completes the proof of $(\mathrm{I})_{m+1}$, and the induction is complete. In particular, Theorem 2.3 is now proven. Theorem 2.5 requires one more step and we leave this to the reader.

5.1. Corollary. Let $m, r \geq 1$ and suppose that $X \subset(0,1)^{m}$ is a definable set. Then there exists a finite set $S$ of functions, each mapping $(0,1)^{\operatorname{dim}(X)}$ to $X$ and of class $C^{(r)}$ such that

(1) $\bigcup_{\phi \in S} \operatorname{range}(\phi)=X$ and

(2) $\left|\phi^{(\alpha)}(\bar{x})\right| \leq 1$ for each $\phi \in S, \alpha \in \mathbb{N}^{\operatorname{dim}(X)}$ with $|\alpha| \leq r$ and all $\bar{x} \in(0,1)^{\operatorname{dim}(X)}$.

Proof. Let $S^{*}$ be an $r$-parameterization of $X$ (as given by Theorem 2.3). Then (1) holds for $S^{*}$ and (2) holds with $c$ in place of 1 , for some $c \in \mathbb{N}$. Cover $(0,1)^{\operatorname{dim}(X)}$ with $(2 c)^{\operatorname{dim}(X)}$ cubes of side $1 / c$ and for each such cube $K$ let $\lambda_{K}:(0,1)^{\operatorname{dim}(X)} \rightarrow K$ be the obvious linear bijection. Then the set of all $\phi \circ \lambda_{K}$ 's, as $\phi$ varies over $S^{*}$ and $K$ over the cover, is the required $S$. The details are left to the reader.

As usual, the existence of definable Skolem functions and a saturation argument imply a uniform version. 
5.2. Corollary. Let $n, m, r \geq 1$ and suppose that $X \subset(0,1)^{n} \times M^{m}$ is a definable family. Then there exists $N \in \mathbb{N}$ and, for each $\bar{y} \in M^{m}$, a set $S_{\bar{y}}$ of $N$ functions, each mapping $(0,1)^{\operatorname{dim}\left(X_{\bar{y}}\right)}$ to $X_{\bar{y}}$ and each of class $C^{(r)}$, such that

(2) $\left|\phi^{(\alpha)}(\bar{x})\right| \leq 1$ for each $\phi \in S_{\bar{y}}, \alpha \in \mathbb{N}^{\operatorname{dim}\left(X_{\bar{y}}\right)}$ with $|\alpha| \leq r$ and all $\bar{x} \in(0,1)^{\operatorname{dim}\left(X_{\bar{y}}\right)}$.

Further, the functions comprising $S_{\bar{y}}$ depend definably on $\bar{y}$.

\section{The "Main Lemma"}

We return to the assumption that $\mathcal{S}$ is an o-minimal structure over $\mathbb{R}$.

By a hypersurface of degree $d$ (in $\mathbb{R}^{n}$ ) we mean a set of the form $\left\{x \in \mathbb{R}^{n}: f(x)=0\right\}$ where $f$ is a nonzero polynomial over $\mathbb{R}$ of degree $d$ in $n$ variables. If $Z \subset \mathbb{R}^{n} \times \mathbb{R}^{m}$ is a family (cf $\S 1$ ), the fibre dimension of $Z$ means the maximum dimension of a fibre of $Z$ (in situations where this makes sense).

The main device in the diophantine part of the argument here, as in $[4,20,22,23]$, is that the rational points of height $\leq T$ in the image of a (sufficiently smooth) map $\phi:[-1,1]^{k} \rightarrow \mathbb{R}^{n}$, where $k<n$, reside on "few" hypersurfaces of prescribed degree $d$ relative to norms of $\phi$ and its derivatives up to some suitable order (depending on $d$ ). A similar result is achieved by $p$-adic means in the algebraic setting in [12].

Already in [4], where $k=1$, the dependence of the estimate on these norms was eliminated by the observation that, for an algebraic or compact analytic curve, the controlled oscillation implies that intervals on which derivatives are "large" have to be "short" and "few". (Another manifestation of "tameness" in [4] is the compactness argument in the proof Theorem 1.) This device has also been used to obtain bounds for the rational points of a pfaff curve in [24].

Here we use the $r$-parameterization results of $\S 2-5$.

6.1. Proposition. Let $k, n \in \mathbb{N}$ with $k<n$. Then there is for each $d \in \mathbb{N}, d \geq 1$ a nonnegative integer $r=r(k, n, d)$ and positive constants $\epsilon(k, n, d), C(k, n, d)$ with the following property.

Suppose $\phi:(0,1)^{k} \rightarrow \mathbb{R}^{n}$ is a function of class $C^{(r)}$ with $\left|\phi^{(\alpha)}(x)\right| \leq 1$ for all $x \in(0,1)^{k}$ and all $\alpha \in \mathbb{N}^{k}$ with $|\alpha| \leq r$. Let $X=\phi\left((0,1)^{k}\right)$ and $T \geq 1$. Then $X(\mathbb{Q}, T)$ is contained in the union of at most

$$
C(k, n, d) T^{\epsilon(k, n, d)}
$$

hypersurfaces of degree $\leq d$. Further, $\epsilon(k, n, d) \rightarrow 0$ as $d \rightarrow \infty$.

Proof. This follows from [22, 4.2], with $r(k, n, d)$ taken to be one more than the $b(k, n, d)$ therein. The constant $c_{16}$ in that result corresponding to $C(k, n, d)$ here depends, in addition to $k, n, d$, on the domain of $\phi$, and the size of the derivatives up to order $r$. So the conditions of the hypothesis on those derivatives and fixed domain mean that here it may be taken to depend only on $k, n, d$. That $\epsilon(k, n, d) \rightarrow 0$ as $d \rightarrow \infty$ is observed just before the proof of [22, 4.2].

6.2. Proposition. ("Main Lemma"). Let $Z \subset(0,1)^{n} \times M^{m}$ be a definable family of fibre dimension $k<n$. Let $\epsilon>0$. There is a $d=d(\epsilon, k, n) \in \mathbb{N}$ and a constant $K(Z, \epsilon)$ with the 
following property. For any $y \in Y$ and $T \geq 1$, the set $X(\mathbb{Q}, B)$, where $X=X_{y}$, is contained in the union of at most

$$
K(Z, \epsilon) T^{\epsilon}
$$

hypersurfaces of degree $\leq d$.

Proof. Take $d$ such that $\epsilon(k, n, d) \leq \epsilon$ and set $r=r(k, n, d)$ as in 6.1. By Corollary 5.2, there is an $N \in \mathbb{N}$ such that, for every $y \in Y$, there is an $r(k, n, d)$-parameterization, $S_{y}$ say, of $X_{y}$ consisting of at most $N$ maps $\phi:(0,1)^{k} \rightarrow \mathbb{R}^{n}$ having all derivatives up to order $r(k, n, d)$ of absolute value bounded by 1 . To each map $\phi \in S_{y}$, by 6.1 , we have that $\phi\left[(0,1)^{k}\right](\mathbb{Q}, T)$ is contained in the union of at most $C(k, n, d) T^{\epsilon}$ hypersurfaces of degree $\leq d$. This establishes the result with $K(Z, \epsilon)=N \cdot C(k, n, d) \cdot \square$

\section{Proof of Theorem 1.10}

If $X \subset \mathbb{R}^{n}$ is definable and $k \leq n$, we denote by $\operatorname{reg}_{\mathrm{k}}(\mathrm{X})$ the subset of $C^{1}$-smooth points of $X$ of dimension $k([9,1.8])$.

\subsection{Proof of $\mathbf{1 . 1 0}$.}

Since the rational points of height $\leq T$ are stable under the maps $x \mapsto \pm x^{ \pm 1}$, as are the algebraic parts of a set, we may suppose that $Z \subset[0,1]^{n} \times \mathbb{R}^{m}$, and so, by a suitable induction on $n$, that $Z \subset(0,1)^{n} \times \mathbb{R}^{m}$.

Consider first the situation in which $A, B, C \subset(0,1)^{n} \times \mathbb{R}^{m}$ are definable sets with $A \cup B=C$. For any $y \in Y_{C}$, it is immediate that $X_{A, y}^{\mathrm{alg}} \cup X_{B, y}^{\mathrm{alg}} \subset X_{C, y}^{\mathrm{alg}}$. Therefore if the theorem holds for $A$ and $B$ and $\epsilon$, with sets $W(A, \epsilon), W(B, \epsilon)$ and constants $c(A, \epsilon), c(B, \epsilon)$ then it holds for $C$ with

$$
W(C, \epsilon)=W(A, \epsilon) \cup W(B, \epsilon), \quad c(C, \epsilon)=c(A, \epsilon)+c(B, \epsilon)
$$

The proof will be by induction on the fibre dimension of $Z$. If the fibre dimension of $Z$ is zero then there is a uniform bound $c$ for the number of points in any fibre, and the theorem holds with $c(Z, \epsilon)=c$. Suppose then that $k>0$ and the theorem holds for all families of fibre dimension $\leq k-1$ and let $Z \subset(0,1)^{n} \times \mathbb{R}^{m}$ be a definable family with fibre dimension $k$, and $\epsilon>0$.

Suppose $k=n$. If $x \in \operatorname{reg}_{\mathrm{k}}(\mathrm{X})$ of any fibre $X$, then $X$ contains an open ball in $\mathbb{R}^{n}$ containing $x$. Therefore $x \in X^{\text {alg }}$. Moreover, for any $k \in \mathbb{N}$, the family

$$
R_{k}(Z)=\left\{\langle x, y\rangle: x \in \operatorname{reg}_{\mathrm{k}}\left(\mathrm{X}_{\mathrm{y}}\right)\right\}
$$

is definable ([9]). Thus the fibres of $A=R_{n}(Z)$ are subsets of the algebraic parts of the fibres of $Z$. So the conclusion for $A$ holds with $W(A, \epsilon)=A$. The fibre dimension of $B=Z-A$ is $\leq k-1$ and so the theorem holds for $B$ by induction. So it may be assumed that $k<n$.

Let $\left\{x_{1}, x_{2}, \ldots, x_{n}\right\}$ be the coordinate system in $\mathbb{R}^{n}$. For a subset $\sigma \subset\{1,2, \ldots, n\}$ let $\Pi_{\sigma}$ denote the linear coordinate subspace of $\mathbb{R}^{n}$ with coordinates $\left\{x_{i}: i \in \sigma\right\}$, and let $\pi_{\sigma}$ be 
the projection of $\mathbb{R}^{n}$ onto $\Pi_{\sigma}$. Let $S=S_{k, n}=\{\sigma \subset\{1,2, \ldots, n\}: \# \sigma=k+1\}$, and put $q=\# S$.

By 6.2, there is $d \in \mathbb{N}$ and a constant $\alpha(Z, \epsilon)$ such that, for any fibre $X$ of $Z$, any subset $\sigma \in S$ and any $T \geq 1,\left(\pi_{\sigma} X\right)(\mathbb{Q}, T)$ is contained in the union of at most

$$
\alpha(Z, \epsilon) T^{\epsilon / 2 q}
$$

intersections of $\pi_{\sigma} X$ with hypersurfaces of degree $\leq d$. (So $X(\mathbb{Q}, T)$ is contained in at most $\alpha(Z, \epsilon)^{q} T^{\epsilon / 2}$ intersections of $X$ with cylinders on hypersurfaces of degree $d$ in each such subspace.)

Let $T \subset \mathbb{R}^{p}$ parameterize real hypersurfaces of degree $d$ in $\mathbb{R}^{k+1}$. (Note that $T=$ $\mathbb{P}^{\nu(k, d)}(\mathbb{R})$, for suitable $\nu$, is compact, so we can take $T \subset[-1,1]^{p} \subset \mathbb{R}^{p}$.) Then

$$
t=\left\langle t_{\sigma}: \sigma \in S\right\rangle \in \prod T_{\sigma} \subset\left(\mathbb{R}^{p}\right)^{q}
$$

corresponds to a choice of a hypersurface $L=L\left(t_{\sigma}\right)$ of degree $d$ in each $(k+1)$-dimensional linear coordinate subspace $\Pi_{\sigma}$ of $\mathbb{R}^{n}$. We have the definable family

$$
\Sigma=\left\{\langle x,\langle y, t\rangle\rangle: \pi_{\sigma}(x) \in L\left(t_{\sigma}\right), \text { all } \sigma \in S\right\} \subset \mathbb{R}^{n} \times\left(\mathbb{R}^{m} \times\left(\mathbb{R}^{p}\right)^{q}\right) .
$$

Consider a fibre $X$ of $\Sigma$. Since any choice of $k+1$ coordinates is algebraically dependent, $X$ is a closed algebraic set in $\mathbb{R}^{n}$ of dimension $\leq k$.

Replace $Z$ by

$$
\left\{\langle x,\langle y, t\rangle\rangle \in \mathbb{R}^{n} \times\left(\mathbb{R}^{m} \times\left(\mathbb{R}^{p}\right)^{q}\right):\langle x, y\rangle \in Z, t \in \prod T_{\sigma}\right\}
$$

which has the same fibres (and so $Z \subset(-1,1)^{n} \times \mathbb{R}^{m+p q}$ ).

The fibre dimension of $Z \cap \Sigma$ is $\leq k$. If

$$
A_{1}=\left\{\langle x,\langle y, t\rangle\rangle \in Z \cap \Sigma: x \notin \operatorname{reg}_{\mathrm{k}}\left(\mathrm{X}_{\mathrm{Z} \cap \Sigma,\langle\mathrm{y}, \mathrm{t}\rangle}\right)\right\}
$$

then the fibre dimension of $A_{1}$ is $\leq k-1$ and, by induction, an estimate

$$
c\left(A_{1}, \epsilon / 2\right) T^{\epsilon / 2}
$$

holds for the number of rational points of height $\leq T$ on a fibre of $A_{1}$ outside (the fibre of) some suitable family $W\left(A_{1}, \epsilon / 2\right)$. This includes in particular the case of an intersection of a fibre $X$ of $Z$ with cylinders on hypersurfaces of degree $d$ when the intersection has dimension $\leq k-1$.

Similarly, the fibre dimension of

$$
A_{2}=\left\{\langle x,\langle y, t\rangle\rangle \in Z \cap \Sigma: x \notin \operatorname{reg}_{\mathrm{k}}\left(\mathrm{X}_{\Sigma,\langle\mathrm{y}, \mathrm{t}\rangle}\right)\right\}
$$

is $\leq k-1$, and an estimate of the above form holds. Likewise for

$$
A_{3}=\left\{\langle x,\langle y, t\rangle\rangle \in Z \cap \Sigma: x \notin \operatorname{reg}_{\mathrm{k}}\left(\mathrm{X}_{\mathrm{Z},\langle\mathrm{y}, \mathrm{t}\rangle}\right)\right\} .
$$


Let then $B$ be the subset of $Z \cap \Sigma$ of points that are regular (of dimension $k$ ) in their fibres in $Z, \Sigma, Z \cap \Sigma$. Consider a point $P=\langle x,\langle y, t\rangle\rangle=\langle x, u\rangle \in B$. In some small neighbourhood $\Delta$ of $x$ in $\mathbb{R}^{n}$, each of the fibres

$$
X_{Z \cap \Sigma, u}, \quad X_{Z, u}, \quad X_{\Sigma, u}
$$

is a $C^{1}$ submanifold of $\mathbb{R}^{n}$ of dimension $k$. Since $X_{Z \cap \Sigma, u} \subset X_{Z, u}, X_{\Sigma, u}$ the sets locally coincide. But the intersection $X_{\Sigma, u} \cap \Delta$ is a semialgebraic set of dimension $k \geq 1$ if $\Delta$ is taken to be a small ball. Therefore $P \in X_{B, u}^{\mathrm{alg}} \subset X_{Z, u}^{\mathrm{alg}}$. The theorem holds for $B$ with $W(B, \epsilon)=B$.

Let now $y \in Y, X=X_{Z, y}, T \geq 1$. Let $P \in X(\mathbb{Q}, T)$. So $\pi_{\sigma}(P) \in\left(\pi_{\sigma} X\right)(\mathbb{Q}, T)$ for any $\sigma \in S$, and therefore lies on one of the hypersurfaces $t_{\sigma}$. So $P$ lies in one of

$$
(\alpha(Z, \epsilon))^{q} T^{\epsilon / 2}
$$

fibres of $Z \cap \Sigma$. Further, either $P$ lies in a family $A_{1}, A_{2}, A_{3}$ for which as estimate

$$
c\left(A_{i}, \epsilon\right) T^{\epsilon / 2}
$$

holds for the number of rational points of height $\leq T$ outside the corresponding fibre of $W\left(A_{i}, \epsilon / 2\right)$, or $P$ lies in $B$. This completes the proof.

7.2. Remark. In the one-dimensional case, application of the method to the function $y=e^{x}$, for which all intersection multiplicities can be precisely controlled, led to natural proofs of (the real versions of) classical transcendence statements [21]. (A similar method was found a little earlier by Laurent [17] (see also [32])). It would be interesting to make the present argument fully quantitative for e.g. the threefold $\log x \log y=\log w \log z, x, y, z, w>0$ associated with the "four exponentials" conjecture, with a view to showing there can be only "few" solutions in some more general sense than the "six exponentials" theorem ([32]).

\section{Dilation-integer points}

We now take $X$ to be a bounded definable set, otherwise the conclusion of 8.1 may fail (e.g. $X=\left\{\left\langle x, 2^{x}\right\rangle, x \in \mathbb{R}\right\}$ definable in $\left.\mathbb{R}_{\text {exp }}\right)$. Note that, for any $X$ and $t,(t X)^{\text {alg }}=t\left(X^{\text {alg }}\right)$.

8.1. Theorem. Let $Z \subset \mathbb{R}^{n} \times \mathbb{R}^{m}$ be a bounded definable family and $\epsilon>0$. There is a definable family $W=W(Z, \epsilon) \subset Z$ and a constant $c(Z, \epsilon)$ with the following property. Let $X=X_{Z, y}$ and put $X_{\epsilon}=X_{W, y}$. Then $X_{\epsilon} \subset X^{\text {alg }}$ and

$$
\#\left(t X-t X_{\epsilon}\right)(\mathbb{Z}) \leq c(Z, \epsilon) t^{\epsilon} .
$$

Proof. Since $Z$ is bounded, say $Z \subset[-C, C]^{n+m}$ where $C>1$, we can assume in fact $Z \subset[-1,1]^{n+m}$ at the cost of replacing the dilation parameter $t$ by $C t$. The proof then follows method of proof of 1.10, using a result for dilation-integer points in the image of a map $\phi:(0,1)^{k} \rightarrow \mathbb{R}^{n}$ with suitably bounded derivatives adapted from $[22,4.1]$ in the same way that 6.1 above adapts $[22,4.2]$ for rational points.

8.2. Remark. In fact only the members of the family $Z$ need be bounded (not the parameters), although there is no real added generality here. 


\section{References}

1. Y. André, Arithmetic Gevrey series and transcendence. A survey, J.Th. Nombres Bordeaux 15 (2003), 1-10.

1. G. Andrews, An asymptotic expression for the number of solutions of a general class of diophantine equations, Trans. Amer. Math. Soc. 99 (1961), 272-277.

3. E. Bierstone and P. D. Milman, Semianalytic and subanalytic sets, Pub. Math. I.H.E.S. 67 (1988), 5-42.

4. E. Bombieri and J. Pila, The number of integral points on arcs and ovals, Duke Math. J. 59 (1989), 337-357.

5. D. Burguet, A proof of Gromov's algebraic lemma, 13 Sep 2005, submitted.

6. L. van den Dries, Remarks on Tarski's problem concerning $(\mathbb{R},+, \cdot, \exp )$, in Logic colloquium '82, Lolli, Longo, and Marcja, editors, North-Holland, Amsterdam, 1984, 97-121.

7. L. van den Dries, Tame topology and o-minimal structures, LMS Lecture Note Series 248 , CUP, Cambridge, 1998.

8. L. van den Dries, A. Macintyre, and D. Marker, The elementary theory of restricted analytic fields with exponentiation, Ann. Math. 140 (1994), 183-205.

9. L. van den Dries and C. Miller, Geometric categories and o-minimal structures, Duke Math. J. 84 (1996), 497-540.

10. A. Gabrielov, Projections of semi-analytic sets, Funct. Anal. Appl. 2 (1968), 282-291.

11. M. Gromov, Entropy, homology and semialgebraic geometry [after Y. Yomdin], Séminaire Bourbaki, 38éme année, 1985-86, exposé 663, Astérisque 145-146 (1987), 225-240.

12. D. R. Heath-Brown, The density of rational points on curves and surfaces, Ann. Math. 155 (2002), 553-595.

13. M. Hindry and J. H. Silverman, Diophantine geometry: an introduction, Graduate Texts in Mathematics 201, Springer, New York, 2000.

14. V. Jarnik, Über die Gitterpunkte auf konvexen Curven, Math. Z. 24 (1926), 500-518.

15. A. G. Khovanskii, Fewnomials, Translations of Mathematical Monographs 88, AMS, Providence, 1991.

16. S. Lang, Number theory III: diophantine geometry, Encyclopedia of Mathematical Sciences, Volume 60, Springer, Berlin, 1991.

17. M. Laurent, Sur quelques résultats récents de transcendance, Journées Arithmétiques 1989 (Luminy, 1989), Astérisque 198-199-200 (1991), 209-230.

18. K. Mahler, Lectures on transcendental numbers, Lecture Notes in Math. 546, Springer, Berlin, 1976.

19. R. S. Phillips and P. Sarnak, The spectrum of Fermat curves, Geom. Funct. Anal. 1 (1991), 80-146.

20. J. Pila, Geometric postulation of a smooth function and the number of rational points, Duke Math. J. 63 (1991), 449-463. 
21. J. Pila, Geometric and arithmetic postulation of the exponential function, J. Australian Math. Soc. Ser. A 54 (1993), 111-127.

22. J. Pila, Integer points on the dilation of a subanalytic surface, Quart. J. Math. 55 (2004), 207-223.

23. J. Pila, Rational points on a subanalytic surface, Ann. Inst. Fourier, 55 (2005), 1501-1516.

24. J. Pila, Note on the rational points of a pfaff curve, Proc. Edin. Math. Soc., to appear.

25. A. J. van der Poorten, Transcendental entire functions mapping every algebraic number field into itself, J. Australian Math. Soc. 8 (1968), 192-193.

26. J.-P. Rolin, P. Speissegger and A. J. Wilkie, Quasianalytic Denjoy-Carleman classes and o-minimality, J. Amer. Math. Soc. 16 (2003), 751-777.

27. P. Sarnak, Torsion points on varieties and homology of abelian covers, unpublished manuscript.

28. P. Sarnak, Diophantine problems and linear groups, Proc. Int. Cong. Math. (Kyoto, 1990), Math. Soc. Japan, Tokyo, 1991, 459-471.

29. W. M. Schmidt, Integer points on curves and surfaces, Monatsh. Math. 99 (1985), 45-72.

30. W. M. Schmidt, Integer points on hypersurfaces, Monatsh. Math. 102 (1986), 27-58.

31. H. P. F. Swinnerton-Dyer, The number of lattice points on a convex curve, J. Number Th. 6 (1974), 128-135.

32. M. Waldschmidt, Diophantine approximation on linear algebraic groups, Grund. Math. Wissen. 326, Springer, Berlin, 2000.

33. A. J. Wilkie, Model completeness results for expansions of the ordered field of real numbers by restricted Pfaffian functions and the exponential function, J.Amer. Math. Soc. 9(1996), 1051-1094.

34. A. J. Wilkie, A theorem of the complement and some new o-minimal structures, Selecta Math. N.S. 5 (1999), 397-421.

35. A. J. Wilkie, Diophantine properties of sets definable in o-minimal structures, J. Symb. Logic 69 (2004), 851-861.

36. Y. Yomdin, Volume growth and entropy, Israel J. Math. 57 (1987), 285-300.

37. Y. Yomdin, $C^{k}$-resolution of semialgebraic mappings. Addendum to Volume growth and entropy, Israel J. Math. 57 (1987), 301-317.

School of Mathematics

University of Bristol

University Walk

Bristol BS8 1TW

UK

j.pila@bristol.ac.uk
Mathematical Institute

University of Oxford 24-29 St Giles' Oxford OX1 3LB

UK

wilkie@maths.ox.ac.uk 